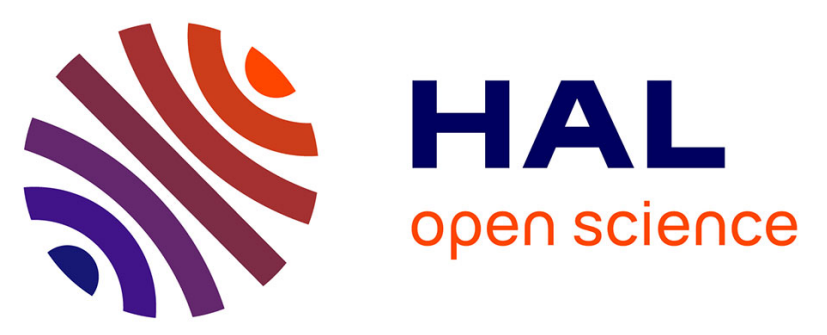

\title{
Le bouddhisme comme fin et moyens de l'armée du Ganden Phodrang: une introduction aux relations des sphères bouddhique et militaire au Tibet (1642-1959)
}

Alice Travers, Federica Venturi

\section{- To cite this version:}

Alice Travers, Federica Venturi. Le bouddhisme comme fin et moyens de l'armée du Ganden Phodrang: une introduction aux relations des sphères bouddhique et militaire au Tibet (1642-1959). Cahiers d'Extrême-Asie, 2019, Le bouddhisme et l'armée au Tibet pendant la période du Ganden Phodrang (1642-1959) / Buddhism and the Millitary in Tibet during the Ganden Phodrang Period (1642-1959), 27 (1), pp.13-22. 10.3406/asie.2018.1504 . hal-02510644

\author{
HAL Id: hal-02510644 \\ https://hal.science/hal-02510644
}

Submitted on 6 Jul 2020

HAL is a multi-disciplinary open access archive for the deposit and dissemination of scientific research documents, whether they are published or not. The documents may come from teaching and research institutions in France or abroad, or from public or private research centers.
L'archive ouverte pluridisciplinaire HAL, est destinée au dépôt et à la diffusion de documents scientifiques de niveau recherche, publiés ou non, émanant des établissements d'enseignement et de recherche français ou étrangers, des laboratoires publics ou privés. 


\section{Le bouddhisme comme fin et moyens de l'armée du Ganden}

\section{Phodrang : une introduction aux relations des sphères}

bouddhique et militaire au Tibet (1642-1959)

\section{Alice Travers, Federica Venturi}

\section{Citer ce document / Cite this document :}

Travers Alice, Venturi Federica. Le bouddhisme comme fin et moyens de l'armée du Ganden Phodrang : une introduction aux relations des sphères bouddhique et militaire au Tibet (1642-1959). In: Cahiers d'Extrême-Asie, vol. 27, 2018. Le bouddhisme et l'armée au Tibet pendant la période du Ganden Phodrang (1642-1959) / Buddhism and the Millitary in Tibet during the Ganden Phodrang Period (1642-1959) pp. 1-11;

doi : https://doi.org/10.3406/asie.2018.1504

https://www.persee.fr/doc/asie_0766-1177_2018_num_27_1_1504

Fichier pdf généré le 07/01/2020 


\title{
LE BOUDDHISME COMME FIN ET MOYENS DE L'ARMÉE DU Ganden Phodrang : \\ UNE INTRODUCTION AUX RELATIONS DES SPHÈRES BOUDDHIQUE et Militaire AU Tibet (1642-1959)
}

\author{
Alice Travers \& Federica Venturi*
}

L'histoire du Tibet, comme celle de tout autre pays, a été marquée par de nombreuses guerres et, de ce point de vue, la période du gouvernement bouddhique des Dalaï-lamas, le Ganden Phodrang (Dga' ldan pho brang, I642-1959)', ne fait pas exception. Cependant, bien que le recours à la guerre dans d'autres civilisations ne surprenne personne, l'idée qu'un gouvernement bouddhique, idéologiquement engagé à ne pas nuire aux êtres vivants, inclut la guerre parmi ses sphères d'activité peut sembler à première vue contre-intuitif. De nos jours encore, le grand public considère le bouddhisme comme étant avant tout une religion de la non-violence ${ }^{2}$ et associe difficilement les Dalaï-lamas et leur gouvernement à l'idée de conflit armé. Le but de ce volume n'est pas tant de dissiper cette idée - qui ne survit pas à une observation même rapide de l'histoire tibétaine -, que de permettre une compréhension plus fine des relations entre les sphères militaire et religieuse au Tibet. Nous avons ainsi rassemblé ici plusieurs études qui explorent les aspects multiples et complexes de ce sujet.

En tant que gouvernement explicitement bouddhique, la protection du bouddhisme, et en particulier de l'école bouddhique à laquelle appartenait le Dalaï-lama, les Gélukpa (Dge lugs pa), était l'objectif ultime de l'État tibétain et de toute action militaire entreprise pour son compte, que ce soit par sa propre armée, par des milices levées temporairement par ce même gouvernement ou bien par des troupes envoyées par les puissances impériales mongoles puis mandchoues. Afin d'atteindre

* Cette introduction est une version révisée et augmentée d'un état de l'art sur les relations entre le bouddhisme et l'armée au Tibet, publié sur le blog du projet TibArmy : Travers (Alice) et Venturi (Federica), «Buddhism, Both the Means and the End of the Ganden Phodrang Army. A State-of-the Field Review on Buddhism vis-à-vis the Military in Tibet », 2017 (https://tibarmy. hypotheses.org/69I, consulté le 25/I0/2017).

I. La période du gouvernement du Ganden Phodrang entre I642 et I959 correspond globalement aux règnes du Cinquième au Quatorzième Dalaï-lamas ou de leurs régents monastiques pendant leur minorité ou pendant les périodes de recherche de leur réincarnation après leur décès. Ces trois siècles ont connu cependant deux brèves périodes se démarquant du cadre institutionnel mentionné car le pouvoir ultime était entre les mains d'un dirigeant laïc, pendant les règnes de Polhané (Pho lha nas) et de son fils (I728-50), et pendant la régence de Shatra Wangchuk Gyelpo (Bshad sgra Dbang phyug rgyal po, r. I862-64).

2. Cette opinion difficile à déconstruire semble survivre, même face à la récente persécution (2017 et 2018) des Rohingya, une minorité musulmane du Myanmar (Birmanie), un pays à prédominance bouddhique. 
ce but, des moines bouddhistes ont souvent joué un rôle de premier plan dans les affaires militaires tibétaines au cours de la période du Ganden Phodrang. De plus, la doctrine bouddhique, et en particulier les rituels de protection et de destruction, étaient utilisés pour soutenir l'action militaire. Dans les sources tibétaines, ces méthodes sont souvent décrites comme ayant joué un rôle déterminant dans l'issue d'un conflit armé.

Cette attitude vis-à-vis de la guerre et de la violence ne constituait pas une exception dans la tradition bouddhique, ni une particularité typique du Tibet, loin des origines de la tradition bouddhique. Au contraire, l'intégration dans le cadre bouddhique de la violence en général, et de la force militaire en particulier, est une caractéristique de cette religion depuis ses origines, comme le montre l'aperçu des études consacrées au thème «bouddhisme et violence » qui suit.

Bien que le bouddhisme ne soit pas une religion normalement associée à la violence, les sociétés bouddhiques n'y sont évidemment pas étrangères, ni même à la violence déclarée et à la guerre, et le Tibet ne fait pas exception. Le premier article scientifique consacré à l'analyse de l'oxymore apparente que représente la " guerre bouddhique » a paru il y a plus de soixante ans, en 1957, sous la plume de l'éminent sinologue Paul Demiéville, sous la forme d'un Postscript à l'Histoire des moines guerriers du Japon du général Gaston Renondeau, intitulé « Le Bouddhisme et la guerre ». La question abordée dans cet article pionnier était de savoir si le phénomène des moines soldats était limité au bouddhisme japonais ou si la militarisation bouddhique sous diverses formes se retrouvait également dans d'autres traditions bouddhiques. Demiéville a utilisé cet article pour explorer les fondements idéologiques utilisés pour justifier la guerre dans des contextes bouddhiques et pour évaluer les raisons possibles de l'apparition du militarisme bouddhique en tant que phénomène historique.

L'auteur a notamment expliqué certains aspects de la théorie éthique bouddhique et de la pratique rituelle à la lumière de leurs relations avec la guerre. Il a observé par exemple que, dans toute l'Asie orientale, des rituels tantriques bouddhiques étaient régulièrement utilisés pour soutenir des objectifs militaires. Il a ensuite souligné que la propagation du bouddhisme n'avait rendu ni la Chine ni le Japon plus pacifistes. Il a toutefois admis que la doctrine bouddhique de la non-violence semblait avoir contribué à l'affaiblissement historique, militairement parlant, du monde « lamaïste » tibéto-mongol. En cela, Demiéville faisait écho à une idée qui reste omniprésente dans le champ des études tibétaines, chinoises et mongoles, ainsi que dans l'opinion publique en général ${ }^{3}$. Il est cependant facile, à la lumière des nombreux exemples de militarisme bouddhiques fournis par les histoires tibétaine et mongole, de voir qu'une telle conception mérite une analyse plus approfondie.

3. Par exemple, en 1928, le diplomate britannique Charles Bell écrivit à propos du Tibet que « [...] le bouddhisme, avec son influence lénifiante interdisant de prendre la vie, a affaibli la puissance martiale de la nation » (notre traduction), cf. Bell (Charles), The People of Tibet, Motilal Banarsidass, New Delhi, 1992, p. 15. Ou bien, l'idée selon laquelle le Tibet serait une "société désarmée" comme l'a décrite George Bataille en 1949, cité dans Faure (Bernard), Bouddhisme et Violence, Le Cavalier Bleu, Paris, 2008, p. 44. 
Dans sa recherche des justifications éthiques bouddhiques du recours à la violence, Demiéville a particulièrement mis en avant ce qu'il a appelé la « justification statistique ", c'est-à-dire l'idée selon laquelle le meurtre peut être justifié si celui d'une personne sauve la vie de nombreuses autres - une éthique utilitaire illustrée par exemple dans l'histoire du capitaine du navire-bodhisattva dans l'Upāyakauśalya Sütra. Il est intéressant de noter que le principal exemple historique sur lequel Demiéville s'appuie pour illustrer ce principe a trait à l'histoire du Tibet, à savoir au meurtre du roi Langdarma (Glang dar ma) en 842 par le moine Lhalung Pelgyi Dorjé (Lha lung Dpal gyi rdo rje). Selon la tradition tibétaine, ce meurtre aurait été provoqué par l'oppression du bouddhisme perpétrée par le roi et son assassin est célébré au Tibet comme un saint. Au moment de se lancer dans son entreprise, Lhalung Pelgyi Dorjé n'aurait nourri que des «pensées de pitié » à l'égard de la future victime.

Pour une raison indéterminée - peut-être en raison d'un déclin de l'intérêt pour l'histoire militaire en général ou en raison de l'attention accrue portée par les études bouddhiques à d'autres aspects de la doctrine et de la pratique - le sujet des relations entre bouddhisme et violence a été presque entièrement négligé par les spécialistes pendant les cinquante ans suivant la parution de l'article de Demiéville. La seule exception fut l'article «Buddhismus und Glaubenskriege » de Lambert Schmithausen, publié en 1996 à l'occasion d'un séminaire consacré au thème des guerres de religion. Cet article aborde de façon systématique quatre questions distinctes mais liées, concernant la guerre et la violence dans le bouddhisme, à savoir I) le « caractère explicite des sources » sur l'existence d'une norme éthique interdisant d'entrer en guerre ; 2) des exemples de violations de cette norme ; 3 ) des «tentatives d'harmonisation de la politique et de l'éthique »; et 4) des «tentatives de relativiser la norme $»^{4}$.

$\mathrm{Au}$ début du $\mathrm{XXI}^{\mathrm{e}}$ siècle, on observe une profusion soudaine de publications consacrées à la relation entre bouddhisme et violence, qui sont parues successivement à un rythme soutenu'. Le premier était une collection d'articles intitulée Buddhism and Violence, éditée par Michael Zimmermann ${ }^{6}$. Ce volume, qui comprend les résultats d'un panel de conférence consacré à ce thème, visait à illustrer le fait que,

4. Schmithausen (Lambert), «Aspects of the Buddhist Attitude towards War », dans Violence Denied: Violence, Non-Violence and the Rationalization of Violence in South Asian Cultural History, édité par Houben (Jan E. M.) et van Kooij (Karel R.), Brill, Leiden, 1999, p. 45-67.

5. Nous ne discutons ici que des travaux qui comportent des parties spécifiquement consacrées au Tibet. D'autres volumes sur ce thème ont paru au cours de la même période en se concentrant sur les contextes de l'Asie du Sud-Est. Parmi ceux-ci, il convient de mentionner les suivants : Tambiah (Stanley J.), Buddhism Betrayed? Religion, Politics and Violence in Sri Lanka, University of Chicago Press, Chicago, 1992 ; Bartholomeusz (Tessa J.), In Defense of Dharma: Just-war Ideology in Buddhist Sri Lanka, Routledge, Londres, 2002 ; Hinnels (John R.) et King (Richard), éds., Religion and Violence in South Asia: Theory and Practice, Routledge, Londres, 2007 ; Jerryson (Michael K.), Buddbist Fury. Religion and Violence in Southern Thailand, Oxford University Press, Oxford et New York, 2oII ; Tikhonov (Vladimir) et Brekke (Torkel), Buddhism and Violence. Militarism and Buddbism in Modern Asia, Routledge, New York, 2013.

6. Zimmermann (Michael), éd., Buddhism and Violence, Lumbini International Research Institute, Lumbini, 2006. 
comme d'autres systèmes religieux, « les traditions du bouddhisme ont activement ou passivement promu des modes de comportement violents ${ }^{7} »$. Il compte huit articles qui vont géographiquement de l'Inde au Japon, en passant par le Tibet. Chronologiquement, ils font appel aux sources les plus anciennes en Pali, à celles plus modernes du Mahāyāna, en allant jusqu'au Xx $x^{\mathrm{e}}$ siècle. Sur le plan thématique, la gamme est également très diverse, avec par exemple une analyse du suicide et de la pratique de l'auto-immolation par le feu - caractéristique des manifestations d'indépendance du Tibet au cours des dernières années - ; une discussion sur les moines soldats au Japon ; et une analyse de la tension éthique vécue par un souverain modèle bouddhiste ou un "souverain universel idéal» (Skt. Cakravartin : litt. « celui qui fait tourner la roue ») pour punir les malfaiteurs.

De manière significative pour le sujet qui nous intéresse, cette collection comprend deux articles consacrés au Tibet, qui traitent tous deux de l'histoire, évoquée ci-dessus, de l'assassinat du roi Langdarma. Le premier, par Carmen Meinert, examine la façon dont le célèbre épisode a été assimilé, dans les sources littéraires tibétaines, au rite tantrique de « libération par le meurtre »; il illustre le fait qu'un acte de meurtre tel que celui de Langdarma, lorsqu'il est perpétré dans un contexte religieux, peut être transformé grâce au rituel, en un acte sacré ${ }^{8}$. Jens Schlieter, de son côté, soutient que ce récit, plutôt que d'illustrer un "meurtre de libération ", vise à fournir une justification du tyrannicide lorsqu'il est effectué dans des circonstances justes ${ }^{9}$. Le récit devient ainsi « un modèle de résolution des conflits politiques ${ }^{\mathrm{IO}}$ ».

Deux ans après la publication du volume d'articles par Zimmermann, Bernard Faure, spécialiste du bouddhisme japonais, publie son ouvrage fondateur Bouddbisme et violence. Il y examine en détails les contradictions et les ambiguïtés inhérentes à la relation entre bouddhisme et violence. Il part du constat qu'il existe un certain décalage entre les idéaux éthiques normatifs du bouddhisme et son approche pragmatique des conflits et de la violence. Tirant des exemples de l'histoire du Sri Lanka, de la Chine, du Vietnam, du Tibet et du Japon, ses analyses englobent non seulement la guerre, mais aussi des exemples de violence symbolique exprimée par la mythologie, l'iconographie ${ }^{\text {II }}$, le rituel et le langage des sermons. Au sein des diverses cultures bouddhiques examinées, il analyse la manière dont le recours à la violence a été légitimé tant sur le plan doctrinal que pratique, et les raisons pour lesquelles les dirigeants bouddhistes pouvaient aisément se permettre de compromettre le principe de non-violence. Les parties de son travail qui synthétisent les débats sociologiques variés sur la relation adéquate entre le bouddhisme et l'État sont particulièrement

7. Ibid., «Introduction », p. 6.

8. Meinert (Carmen), «Between the Profane and the Sacred? On the Context of the Rite of "Liberation" (sgrol ba)", dans Ibid., p. 99-I30.

9. Schlieter (Jens), "Compassionate Killing or Conflict Resolution? The Murder of King Langdarma according to Tibetan Buddhist Sources ", dans Ibid., p. I3I-58.

Io. Ibid., «Introduction », p. 8.

II. On pense ici, pour le Tibet, à l'article d'Amy Heller « Armor and Weapons in the Iconography of Tibetan Buddhist Deities ", dans LaRocca (Donald J.), Warriors of the Himalayas. Rediscovering the Arms and Armor of Tibet, The Metropolitan Museum of Art, Yale University Press, New York, 2006, p. 35-42. 
stimulantes pour notre propre recherche sur la guerre pendant la période du Ganden Phodrang. En effet, Faure souligne le fait que tout État, bouddhique ou non, dans la mesure où il détient par définition le monopole de l'usage légitime de la violence, ne peut renoncer à ce monopole sans perdre en même temps son droit de gouverner et sa capacité à maintenir l'ordre ${ }^{12}$. Dans ce contexte, il souligne l'humanisme de la religion bouddhique - une religion construite par les humains pour servir les humains - et le fait que les humains ne parviennent pas toujours à respecter les idéaux qu'ils se sont eux-mêmes créés. De son point de vue, étant donné que les sociétés bouddhiques ont été contraintes par la force des circonstances à tolérer la violence de la part des États, le fait même que l'idée de non-violence se soit maintenue au niveau d'un idéal reste un acquis significatif à porter à leur crédit ${ }^{13}$.

The Taming of the Demons de Jacob Dalton s'adresse spécifiquement au discours sur la violence dans le bouddhisme tibétain et en particulier au rituel tantrique de "libération » ou de meurtre ${ }^{14}$. En analysant les preuves textuelles de divers genres littéraires et de diverses périodes historiques, Dalton reconstruit ce qu'il appelle une « histoire de la violence » dans le bouddhisme tibétain. Dans une perspective parallèle, le livre de Nicolas Sihlé, Rituels bouddbiques de pouvoir et de violence ${ }^{15}$, examine la figure du tantriste tibétain, associée à des rituels visant à tuer, par le biais de pratiques d'exorcisme et de sorcellerie. Le travail porte sur la violence des rituels d'exorcisme et la tension qu'elle crée dans l'activité de spécialistes religieux bouddhistes à Baragaon (Mustang, Népal). Cependant, ces travaux sont essentiellement axés sur la violence et la sorcellerie rituelles, plutôt que sur la violence littérale ou la guerre, mais, comme Bernard Faure l'a souligné, il n'y a pas de discontinuité dans les relations sociales pré-modernes entre la violence symbolique telle qu'on la trouve dans les rituels et la violence physique ${ }^{16}$.

De fait, la violence symbolique fait effectivement partie de l'histoire militaire tibétaine, car des moyens rituels ont été très fréquemment employés pour soutenir des campagnes militaires et des objectifs politiques, la réputation d'efficacité de tels rituels constituant un atout important pour les chefs religieux. Les exemples sont trop nombreux pour être cités ici, et le sujet de la «magie de guerre » est au cœur de l'un des chapitres de ce volume (voir la contribution de George FitzHerbert). Il se trouve que jusqu'à ce jour, la plupart des érudits s'étaient intéressés à de telles pratiques au cours de l'histoire tibétaine précédant le Ganden Phodrang, par exemple avec une figure religieuse du XII ${ }^{\mathrm{e}}$ siècle, Lama Zhang (Bla ma Zhang, II22-93) ${ }^{\mathrm{r}}$, ou le spécialiste rituel Sokdokpa Lodrö Gyeltsen (Sog bzlog pa Blo gros rgyal mtshan,

I2. Faure (Bernard), op. cit., p. 48.

I3. Ibid., p. I6.

I4. Dalton (Jacob), The Taming of the Demons: Violence and Liberation in Tibetan Buddhism, Yale University Press, New Haven, 20II.

15. Sihlé (Nicolas), Rituels bouddhiques de pouvoir et de violence, Brepols, Turnhout, 2013.

I6. Faure (Bernard), Unmasking Buddhism, Wiley-Blackwell, Malden, 2009, p. 95, cité dans Sihlé (Nicolas), op. cit., p. II.

I7. Yamamoto (Carl), Vision and Violence: Lama Zhang and the Politics of Charisma in Twelfth-Century Tibet, Brill, Leiden, 2012. 
I552-I624 ${ }^{18}$. Ils avaient également examiné des pratiques de ce type dans des territoires tibétains non contrôlés directement par le gouvernement Ganden Phodrang ou son armée, comme par exemple l'utilisation bien connue de la "magie de guerre » lors des campagnes Qing dans les régions frontalières tibétaines de Gyelrong (Tib. Rgyal rong; Ch. Jinchuan) en $177 \mathrm{I}^{-} 76^{19}$.

Dans l'anthologie d'articles dirigée par Jerryson et Juergensmeyer et intitulée Buddhist Warfare ${ }^{20}$, l'accent n'était pas mis sur la violence en général, mais sur la guerre en particulier. Le but explicite de ce volume, qui inclut notamment une traduction anglaise de l'article de Demiéville évoqué ci-dessus, était de « perturber l'imaginaire social qui tient les traditions bouddhiques pour exclusivement pacifistes $»^{21}$. En accord avec cet objectif provocateur, la couverture du livre montre un jeune novice bouddhiste tenant ce qui ressemble à un pistolet (une image qui n'a pas été bien reçue par certains cercles bouddhiques de l'Ouest) ${ }^{22}$. À l'instar du recueil édité par Zimmermann, cette publication présente une vaste gamme de données, tant sur le plan géographique que temporel, illustrant le fait que la guerre dans un contexte bouddhique n'était pas confinée à une période ou à un lieu spécifique. Ce volume illustre la façon dont les écritures bouddhiques peuvent être interprétées pour justifier la guerre ${ }^{23}$. Par exemple, l'exploration de l'Ārya-Satyakaparivarta Sütra par Stephen Jenkins conclut finalement que certains discours canoniques du Bouddha suggèrent non seulement que la guerre peut être alléguée, mais qu'il est également possible dans certaines circonstances d'accumuler du mérite par la guerre. Dans les Écritures, soutient-il, le dharma ne donne pas seulement le droit de gouverner à un cakravartin, il lui permet également de faire respecter son règne par la violence.

Le volume de Zimmermann comprend également un article sur le Tibet qui est particulièrement pertinent pour le thème de notre numéro thématique, car il traite de la période de formation de l'État du Ganden Phodrang à l'époque du Cinquième Dalaï-lama Lozang Gyatso (Blo bzang rgya mtsho, I6I7-82). Dans cette

I8. Gentry (James), « Representations of Efficacy: The Ritual Expulsion of Mongol Armies in the Consolidation and Expansion of the Tsang (gTsang) Dynasty ", dans Tibetan Ritual, édité par Cabezón (José), Oxford University Press, Oxford et New York, 2009 ; et, du même auteur, Power Objects in Tibetan Buddhism: The Life, Writings, and Legacy of Sokdokpa Lodrö Gyeltsen, Brill, 2017 .

I9. Martin (Dan), «Bonpo Canons and Jesuit Cannons: On Sectarian Factors Involved in the Ch'ien Lung Emperor's Second Gold Stream Expedition of I77I to 1776 Based Primarily on Some Tibetan Sources ", The Tibet Journal, I990, I5, n² 2, p. 3-28; Waley-Cohen (Joanna), The Culture of War in China. Empire and the Military under the Qing Dynasty, IB Tauris, Londres, New York, 2007.

20. Jerryson (Michael K.) et Juergensmeyer (Mark), éds, Buddhist Warfare, Oxford University Press, Oxford et New York, 20io.

2I. Ibid., p. 3.

22. Plusieurs blogs bouddhistophiles sur Internet ont critiqué le thème abordé dans le livre et même son titre, accusant les rédacteurs en chef et les auteurs d'adopter une position antibouddhiste. En outre, il a été demandé si la photographie avait été mise en scène. Voir par exemple http:// mybuddhaispink.blogspot.com/20II/o3/buddhist-warfare-introduction.html\#comment-form, consulté le 9 juillet 2017 .

23. Jerryson (Michael K.) et Juergensmeyer (Mark), op. cit., p. 5 . 
étude, Derek Maher analyse la façon dont les guerres des années i630 et I640, qui ont abouti à l'établissement officiel de l'État de Ganden Phodrang en I642, ont été justifiées de différentes manières par le Cinquième Dalaï-lama lui-même dans ses divers écrits ${ }^{24}$. Maher examine à la fois l'histoire du Tibet rédigée par le Cinquième Dalaï-lama, intitulée le Chant de la reine du printemps et composée peu de temps après les événements décrits (I643), et les trois premiers volumes de La fine étoffe de soie, son autobiographie composée vingt-cinq ans plus $\operatorname{tard}^{25}$. Maher note en particulier que, dans son histoire, la violence de la guerre qui l'a amené au pouvoir n'était ni dissimulée ni occultée, et qu'il était clairement préoccupé par la justification de cette violence. L'ennemi y est décrit comme étant sous l'emprise d'influences démoniaques, tandis que Gushri Khan (1582-1655), le chef mongol qui a mené ces guerres pour le compte du Dalaï-lama et de son école bouddhique, est félicité pour son altruisme dans la protection et la promotion de la cause bouddhique, qui le rend semblable à un bodhisattva. Gushri Khan est identifié comme étant une émanation de Vajrapāṇi (le bodhisattva considéré comme la manifestation de la puissance de tous les bouddhas) et est décrit comme un « second Songtsen Gampo » (Srong btsan sgam po, c. 605-649), le célèbre empereur tibétain du VII ${ }^{\mathrm{e}}$ siècle. Il est également honoré du titre de dharmarāja ou roi selon le dharma. De cette manière, le Dalaï-lama célèbre de façon emphatique et non équivoque la violence perpétrée au nom du bouddhisme en identifiant Gushri Khan à un bodhisattva et ses opposants à des démons ${ }^{26}$.

En revanche, dans l'autobiographie, rédigée bien plus tard lorsque la position de pouvoir du Grand Cinquième était beaucoup plus assurée, ces mêmes conflits sont décrits un peu différemment. Il y suggère sa propre réticence ou même son opposition à ces guerres et les décrit comme le résultat d'une série de réactions à des circonstances indésirables : à savoir les obstructions, les intimidations et les harcèlements répétés que les Bönpo (Bon po) et les Kagyüpa (Bka’ brgyud pa), deux écoles religieuses du Tibet, avaient infligé aux Gélukpa, l'école des Dalai-lamas, au cours des décennies précédentes. Ainsi, le Cinquième Dalaï-lama valide l'utilisation de la force militaire de manière plus subtile : il s'agit d'une réponse malheureuse mais nécessaire au comportement immoral de ses adversaires ${ }^{27}$.

24. Maher (Derek), «Sacralized Warfare: The Fifth Dalai Lama and the Discourse of Religious Violence », dans Jerryson (Michael K.) et Juergensmeyer (Mark), éds, op. cit., p. 77-90.

25. Karmay (Samten), The Illusive Play. The Autobiography of the Fifth Dalai Lama, Serindia Publications, Chicago, 20I4, p. 2-3, où il est expliqué que le Cinquième Dalaï-lama, bien qu'encouragé à écrire une autobiographie dès I644, n'a entrepris cette tâche qu'en I667. Le troisième volume de son autobiographie, qui concerne les années i675 à I68I, a été composé au cours des dernières années de sa vie, donc de façon presque concomitante avec les événements relatés, puisque le récit n'a pu être commencé avant 1675 et s'est achevé avant la mort du Dalaïlama, intervenue pendant le deuxième mois de 1682 .

26. Maher (Derek), op. cit., p. 8I.

27. Maher (Ibid.) affirme que, dans son autobiographie, le Cinquième Dalaï-lama «menait le dernier combat de cette guerre en plaçant le conflit récemment conclu dans un cadre qui le rendait significatif, qui l'exonérait et justifiait son autorité »(notre traduction). Il ajoute plus tard que le Cinquième Dalaï-lama, dans les trois premiers volumes, et Dési Sanggyé Gyatso (Sde 
Si Demiéville peut être tenu pour le père de la recherche académique sur les relations entre le bouddhisme et la violence en général, Donald LaRocca peut être considéré comme le père de la recherche sur le bouddhisme et la culture matérielle de la guerre au Tibet en particulier. En 2006, il publia une étude historique sur les armes et les armures tibétaines du $\mathrm{VII}^{\mathrm{e}}$ au XX $\mathrm{XX}^{\mathrm{e}}$ siècles, comme catalogue d'une exposition présentée au Metropolitan Museum of Art de New York ${ }^{28}$. Ce volume documente la présence significative d'armes et d'armures au Tibet et leur évolution au cours des siècles, et particulièrement pendant la période du Ganden Phodrang, et il comprend le premier lexique de terminologie dans ce domaine ${ }^{29}$.

Cependant, l'étude la plus novatrice et la plus complète à ce jour sur l'histoire militaire du Tibet est l'œuvre de Gyaltse Namgyal Wangdue (1976) 30, avec sa réimpression d'une version augmentée en $2003^{31}$ sous l'égide de l'Association des vétérans de l'armée tibétaine (Bod dmag rnying pa'i skyid sdug). Il s'agit d'une mine d'informations sur l'armée tibétaine du Ganden Phodrang, car elle contient de nombreux témoignages de première main de l'auteur lui-même, qui a servi comme soldat, et de plusieurs anciens fonctionnaires et officiers de l'armée tibétaine, comme Taring ('Phreng ring) et Nornang (Nor nang). Néanmoins, l'œuvre dans son ensemble a un statut hybride entre une source primaire et une source secondaire : des passages entiers sont parfois reproduits de l'histoire du Tibet écrite par Shakabpa (I907-89), membre du gouvernement du Ganden Phodrang et historien ${ }^{32}$, ou de sources en langue tibétaine, et parfois l'auteur fournit de nouvelles informations sans citer la source originale, ce qui limite l'utilité de l'œuvre.

srid Sangs rgyas rgya mtsho, $1653^{-1705}$ ) dans les trois derniers, avaient en fait tenté d'édulcorer le rôle joué par le Dalaï-lama dans le versement de sang qui avait présidé à l'établissement de son pouvoir : «Du point de vue confortable de cette mythologie pleinement articulée - et à ce moment-là couronnée de succès -, ils semblent avoir décidé de soustraire le Dalaï-lama de toute responsabilité dans les guerres des années I630 et I640, en niant qu'il ait approuvé le bain de sang le plus important qui soit. Il se peut également que, à ce moment-là, le Dalaï-lama, alors âgé, ait fini par avoir des doutes sur les violences déclenchées en son nom. Il est évident, par exemple, que le Dalaï-lama est resté troublé par l'impact humain des combats. Un grand nombre de rêves et de visions rapportés dans la biographie secrète du Cinquième Dalaï-lama montre qu'il était souvent perturbé par des spectres de violence et de guerre » (notre traduction).

28. LaRocca (Donald J.), op. cit.

29. Il a plus tard continué à développer ce thème, cf. «Tibetan Warriors: The Challenges of Presenting the Warlike Side of a Peaceful Culture », dans Challenges and Choices in a Changing World: Proceedings of the ICOMAM Conference, Vienna 2007, Heeresgeschichtliches Museum, Vienna, 2008, p. 39-52.

30. Dwang slob 'go 'dzin rgyal rtse rnam rgyal dbang 'dud [sic], Bod ljongs rgyal kbab chen po'i srid lugs dang 'brel ba'i drag po'i dmag gi lo rgyus rags bsdus, Tibetan Cultural Printing Press, Dharamsala, 1976.

3I. Dwang slob mda' zur spyi 'thus rgyal rtse rnam rgyal dbang'dus, Bod rgyal khab kyi chab srid dang 'brel ba'i dmag don lo rgyus, 2 vols, Bod dmag rnying pa'i skyid sdug, Dharamsala, 2003. L'ouvrage fut finalement traduit en anglais : Gyaltse Namgyal Wangdue (trad. Yeshi Dondup), Political and Military History of Tibet, 2 vols., LTWA, Dharamsala, 2010 et 2012.

32. Shakabpa (Tsepon Wangchuk Deden), Bod kyi srid don rgyal rabs, 2 vols., Shakabpa House, Kalimpong, 1976. 
Comme autres contributions importantes à l'étude des affaires militaires pendant la période du Ganden Phodrang, citons une enquête de Heather Stoddard sur l'utilisation de rituels destinés à repousser l'ennemi ( $d m a g$ bzlog) lors de la guerre anglo-tibétaine de $1888^{33}$; un article d'Elliot Sperling traitant de la représentation du Tibet comme terre de paix non touchée par la guerre ou la violence ${ }^{34}$; et enfin, quelques études rédigées par nous-mêmes - les éditrices de ce volume. Alice Travers a publié des articles sur des laïcs aristocratiques ayant servi comme officiers de l'armée au début du $\mathrm{Xx}^{\mathrm{e}}$ siècle $^{35}$, sur le droit militaire ${ }^{36}$, et le développement de l'institution militaire tel qu'il apparaît dans les documents juridiques du $\mathrm{XVII}^{\mathrm{e}}$ au $\mathrm{XX}^{\mathrm{e}}$ siècle $^{37}$. Cet article représentait une tentative d'identifier les lacunes existant dans notre compréhension de l'évolution de l'armée du Ganden Phodrang afin de définir le potentiel et la direction de ses recherches actuelles sur l'histoire institutionnelle et sociale de l'armée tibétaine.

Dans son article de 20I4, Federica Venturi a mis en lumière l'implication personnelle du Treizième Dalaï-lama Thubten Gyatso (Thub bstan rgya mtsho, I876-I933) dans les affaires militaires tibétaines, ainsi que les idées personnelles du hiérarque concernant l'utilité de l'armée, en traduisant quatre documents qui confirment sans équivoque l'implication directe de l'institution monastique, à l'échelon le plus élevé, dans les affaires militaires tibétaines ${ }^{38}$. Ces documents montrent que le Treizième Dalaï-lama était personnellement impliqué dans la gouvernance temporelle du Ganden Phodrang et qu'il était parfaitement conscient des pressions extérieures et des défis de la realpolitik auxquels le Tibet était confronté à l'époque. Ces menaces

33. Stoddard (Heather), «The Great "Phi gling dmag zlog" of i888. The First Hands-On Confrontation between Tibet and the British Raj with the Participation of Leading Lineage Holders of the "I90o Sngag mang Phur thog gos dkar lcang lo can", Lay mantrins of Reb kong, Amdo, in the Dga' ldan Pho brang State Military Ritual to "Turn Back the Philing” Foreigners ». Article non publié, présenté lors de la $\mathrm{II}^{\mathrm{e}}$ Conférence de l'International Association for Tibetan Studies, 27 août-2 septembre 2006, Bonn.

34. Sperling (Elliot), " "Orientalism" and Aspects of Violence in the Tibetan Tradition », dans Imagining Tibet. Perceptions, Projections and Fantasies, édité par Dodin (Thierry) et Räther (Heinz), Wisdom Publications, Boston, 200I, p. 317-29.

35. "The Horse-Riding and Target-Shooting Contest for Lay Officials (drung 'khor rtsal rgyugs): Reflections on the Military Identity of the Tibetan Aristocracy at the Beginning of the $20^{\text {th }}$ Century ", Études mongoles et sibériennes, centrasiatiques et tibétaines, 42, $201 \mathrm{II}$ (https://emscat. revues.org/I850).

36. "The Lcags stag dmag khrims (1950): A New Development in Tibetan Legal and Military History? ", dans Social Regulation: Case Studies from Tibetan History, édité par Bischoff (Jeannine) et Mullard (Saul), Brill, Leiden, 20I6, p. 99-I25.

37. "The Tibetan Army of the Ganden Phodrang in Various Legal Documents $\left(17^{\text {th }}-2 \mathrm{O}^{\text {th }}\right.$ Centuries) ", dans Secular Law and Order in the Tibetan Highland. Contributions to a Workshop Organized by the Tibet Institute in Andiast (Switzerland) on the Occasion of the $65^{\text {th }}$ Birthday of Christoph Cüppers from the $8^{\text {th }}$ of June to the I2 ${ }^{\text {th }}$ of June 20I4, édite par Schuh (Dieter), Monumenta Tibetica Historica, Abteilung III, Band I3, IITBS GmbH, Andiast, p. 249-66.

38. "The Thirteenth Dalai Lama on Warfare, Weapons and the Right to Self-Defense ", dans Trails of the Tibetan Tradition. Papers for Elliot Sperling, édité par Vitali, Roberto, Amnye Machen Institute, Dharamsala, 20I4, p. 483-509. 
nécessitaient, selon lui, des solutions pragmatiques telles que la modernisation de l'armée, qui devaient prévaloir sur les réserves éthiques bouddhiques.

Les six articles de ce volume constituent une première tentative d'examiner la question générale des relations entre bouddhisme et violence dans le contexte de l'histoire militaire du Ganden Phodrang sous différents angles. Les questions évoquées plus haut, à savoir les différentes élaborations discursives et éthiques utilisées pour légitimer le recours à la violence, la justification de la guerre comme nécessaire à la protection d'un gouvernement bouddhique, l'implication des moines dans les conflits et des soldats dans les affaires religieuses, tous ces thèmes apparaissent de différentes manières dans les recherches présentées ici, que nous avons organisées chronologiquement afin de fournir une image diachronique du développement des relations entre les sphères bouddhique et militaire.

Les deux premiers articles se penchent sur la période initiale et fondatrice du Ganden Phodrang, à savoir le règne du Cinquième Dalaï-lama (I642-82), complétant l'étude séminale de Maher sur cette période, que nous avons mentionnée plus haut. George FitzHerbert montre le rôle déterminant joué par les rituels dans l'idéologie politique et militaire, en étudiant un ensemble de rites destinés à favoriser une issue favorable au combat, et en montrant la place cruciale que cette « magie de guerre » occupait dans la tradition bouddhique tibétaine et dans la production littéraire du Cinquième Dalaï-lama en particulier. Se focalisant sur la même période, Federica Venturi analyse d'autres écrits du Cinquième Dalaï-lama, cette fois-ci dans le but d'évaluer l'implication de ce hiérarque bouddhiste dans les diverses activités militaires de son règne. Elle conclut que les interventions militaires sanctionnées par le Ganden Phodrang se sont produites assez fréquemment durant cette période, et que le Cinquième Dalaï-lama était généralement conscient des situations qui ont conduit au conflit et connaissait bien les détails des préparatifs militaires.

Le troisième article est consacré au XVIII ${ }^{\mathrm{e}}$ siècle et au rôle que les hiérarques bouddhistes peuvent jouer dans l'historiographie tibétaine comme témoins des conflits militaires. Marlene Erschbamer illustre ici l'effet de la guerre contre les Gurkha (I788-9I) sur un maître de la tradition Barawa ('Ba' ra ba ; une école Kagyü), qui se trouvait sur le champ de bataille dans la région de Kyirong (Skyid grong) peu après un conflit armé, et qui a consigné ses impressions dans son autobiographie. Comme une large part des sources historiographiques disponibles au Tibet se compose de biographies religieuses (rnam thar), cette analyse ouvre la porte à d'autres recherches sur des événements similaires dans d'autres hagiographies.

Les trois derniers articles sont consacrés à la dernière période du Ganden Phodrang, la première moitié du $\mathrm{xx}^{\mathrm{e}}$ siècle. Alice Travers examine la participation concrète de certains moines comme acteurs, et non plus comme simples spectateurs, aux activités militaires menées par le gouvernement du Ganden Phodrang : elle analyse le rôle des fonctionnaires ecclésiastiques (rtse drung), employés communément dans l'administration militaire comme secrétaires et responsables de la paie militaire, et comme Commandants-en-chef des armées tibétaines, mais aussi comme officiers d'active à la tête de troupes sur le champ de bataille, et elle s'interroge sur les implications idéologiques de cette organisation. L'article de Ryōsuke Kobayashi étudie la participation de certains monastères situés au 
Kham (Khams) à des conflits militaires impliquant les gouvernements tibétains et chinois dans le premier quart du $\mathrm{Xx}^{\mathrm{e}}$ siècle, et présente le cas de la militarisation du monastère de Dargyé (Dar rgyas). Son article fournit une illustration du fait que le plurilinguisme (chinois et tibétain) des traités bilatéraux permettait, grâce à la différence effective des textes eux-mêmes, une divergence d'interprétation de ces traités par les différentes parties. Enfin, Stacey Van Vleet étudie un nouveau développement, qui prend place également dans cette période, celui de la naissance d'une forme de « médecine militaire » tibétaine, la tradition médicale tibétaine étant elle-même fondamentalement enracinée dans les principes bouddhiques. L'Institut de médecine et d'astrologie ou Mentsikhang (Sman rtsis khang), créé par le Treizième Dalaï-lama en 19ı6, formait déjà des médecins-astrologues qui pouvaient être envoyés auprès de chefs militaires tibétains ; une nouvelle étape est franchie sous la régence de Réting Thupten Jampel Yéshé Gyeltsen (Rwa sgreng Thub bstan 'jam dpal ye shes rgyal mtshan, r. 1934-4I) lorsque trente à quarante soldats de l'armée permanente tibétaine sont admis au Mentsikhang pour y suivre le cursus traditionnel de médecine et astrologie.

Ainsi, ce volume montre que les projets religieux et militaires se sont constamment soutenus pendant la période du Ganden Phodrang. Le bouddhisme fournissait à l'armée à la fois une fin - la protection du gouvernement bouddhique - et des moyens - qu'ils soient humains par le biais de la participation permanente de moines à l'institution militaire et de l'organisation de rituels, ou que ces moyens soient discursifs et philosophiques, lorsqu'il fallait justifier le recours à la violence. 